\title{
La lectura documentaria como disciplina curricular en el curso de biblioteconomía: contenido y metodologías del abordaje socio-cognitivo de la metacognición del indizador aprendiz
}

Documentary reading as curricular discipline in Librarianship study: content and methodologies of social-cognitive approach to metacognition of the apprentice indexer

\section{Mariângela Spotti Lopes Fujita}

(1) Universidad Estadual Paulista Júlio de Mesquita Filho - UNESP, Departamento de Ciencia de Información, Av. Hygino Muzzi Filho, 737, Campus Universitário, CEP. 17525900 - Marília, SP - Brasil, Caixa-Postal: 421, fujita@marilia.unesp.br

\begin{abstract}
Resumen
Se indica la necesidad de presentar a los Cursos de Graduación la propuesta de adecuación y/o inclusión del contenido programático, metodologías de enseñanza y bibliografía que se refieran a la lectura documentaria para análisis de asunto. En la disciplina "Lectura Documentaria", fue adoptado el plan de enseñanza recomendado por las directrices del Protocolo sumado a un plan de aulas que permite la práctica pedagógica de la lectura documentaria mediante fundamento teórico y el uso del Protocolo Verbal. Se observó una disminución de las dificultades de análisis de asunto practicado en la disciplina "Indexación". Es necesario que los currículos orientados a la formación de indizadores puedan incluir la disciplina de "Lectura Documentaria" o adecuar contenidos programáticos.
\end{abstract}

Palabras clave: Lectura Documentaria. Indización. Protocolo Verbal.

\section{Introducción}

En el análisis de asunto, el indizador tiene como tarea la extracción de contenidos significativos del contenido textual expresado por términos que, una vez aislados del contexto del texto, deben representarlo de tal forma que un usuario, en una situación de búsqueda de aquel determinado asunto, en cualquier momento, pueda recuperar el texto por medio de esos mismos términos, estableciendo, por lo tanto, una correspondencia de relevancia entre el significado del contenido textual recuperado y el significado atribuido por el usuario en el momento de la búsqueda. En esa rápida explanación segmentamos el procedimiento del análisis de asunto (extracción de conceptos significativos) de su

\begin{abstract}
It is outpointed the need of presenting, to the undergraduated courses, a proposal of adequacy and/or inclusion of programmatic content, teaching methodologies and bibliography relating to the documentary reading for subject analysis. It was adopted a teaching plan recommended by the guidelines of the program, in addition to a syllabus that allows for the pedagogical practice of documentary reading through theoretical basis and the use of the Verbal Protocol. It was observed the decreasing of the difficulties in subject analysis, practiced in the "Indexing" discipline. It is necessary that the curricula devoted to indexers' education can include the "Documentary Reading" discipline or adequate programmatic contents.
\end{abstract}

Keywords: Documentary reading. Indexing. Verbal Protocol.

objetivo (representar para recuperar) para dejar claro que el objetivo de la lectura profesional del indizador para análisis de asunto es la extracción de conceptos significativos del contenido textual.

Por realizarse en el análisis de asunto, fase inicial de la indexación, la lectura necesita de condiciones especificas para el desarrollo de ese proceso, como el conocimiento previo y estrategias construidas y aprendidas durante toda la vida.

De esa forma, como principales contribuciones para la tarea, podemos resaltar el ofrecimiento de otra perspectiva teórico-práctica de la comprensión del proceso de indexación y de las dificulta- 
des de un indizador frente a un documento, observando el proceso de lectura para identificar las estrategias de comprensión que pretenden la identificación y selección de conceptos.

La lectura es un proceso que permite el análisis de asunto de textos, influyendo en el resultado de la indexación. El estudio de la lectura por medio de la interacción de sus variables -el texto, el lector y el contexto- (Fujita, 2003, 2004) pretende comprender las dificultades de análisis de asunto de textos y reunir apoyos para la orientación de la formación del indizador en lectura documentaria que contribuyan para el perfeccionamiento de la capacidad de lectura del indizador.

La propuesta de un Programa de orientación para la formación del indizador en lectura documentaria, a partir de los resultados obtenidos, se basa en el abordaje y organización de los apoyos teóricos y metodológicos para que sea posible, no solo la comprensión, sino también la articulación coherente de todos los conocimientos en una disciplina obligatoria para el currículo de Biblioteconomía. La planificación y ejecución del Programa consta de una propuesta de contenido programático sobre lectura en análisis documentario para indexación, acompañada de bibliografía y metodologías de enseñanza, dando origen a una disciplina.

De esa forma, el trabajo tiene el objetivo estratégico de presentar la propuesta del plan de enseñanza de la disciplina obligatoria "Lectura documentaria", realizada en el Curso de Graduación de Biblioteconomía de la UNESP Campus de Marília en los años de 2005 y 2006, conteniendo la sistematización y ordenación secuencial de los contenidos, acompañados de recomendaciones metodológicas en lo que se refiere al uso de recursos pedagógicos que propician la enseñanza con un abordaje cognitivo y socio-cognitivo.

\section{La disciplina Lectura Documentaria en el Programa de Orientación para la formación del indizador}

En las Directrices del Programa de orientación constan los principales apoyos teóricos y metodológicos del estudio que servirán de base a la creación y perfección de la enseñanza de indexación. De esa forma, el Programa de orientación se constituye de tres partes: la parte conceptual sobre lectura en análisis documentario, conteniendo el fundamento teórico-práctico a partir de los resultados de investigación, una parte metodológica dedicada al modelo de lectura documentaria para indexación y la parte de recursos pedagógicos para efectuar un abordaje cognitivo y socio-cognitivo en el aula.

\section{A) Parte conceptual}

Los apoyos más importantes colectados se refieren a los siguientes aspectos: lectura y estrategias de lectura; el texto y su estructura; el indizador como lector: sus estrategias y conocimiento previo; y el lector profesional y su contexto. La organización de esos apoyos se moderó, por la perspectiva interaccionista de las variables que participan del proceso de lectura el texto, el lector y el contexto-, produciendo la estructura básica: lectura en análisis documentaria; texto -estructura textual en la lectura documentaria-; lector -el indizador como lector profesional-; contexto -la indexación en sistemas de información-.

En la variable texto, el conocimiento previo de la estructura textual permite al indizador identificar el contenido informacional.

En la variable lector, el análisis de su actuación profesional revela que a pesar de la experiencia en indexación posee dificultades en la identificación de conceptos, momento en que interactúa con el texto por medio de la lectura. El análisis de su formación profesional indica que fue preparado para un análisis de asuntos orientado por el uso de lenguajes documentarios.

En la variable contexto, el conocimiento de la situación comunicativa y de sus reglas permitió el conocimiento del contexto de trabajo desarrollado por indizadores en sistemas de información: los objetivos de la indexación, la política de indexación, las reglas y procedimientos del manual de indexación, el lenguaje documentario y los intereses de búsqueda del usuario.

\section{B) Parte metodológica}

El aspecto más contundente de la observación de la lectura documentaria es que indizadores, bibliotecarios y especialistas no demostraron un dominio de procedimientos sistemáticos para abordaje del contenido textual para la identificación de conceptos. Esta constatación permitió la posibilidad de un uso combinado de la exploración de la estructura textual con el cuestionamiento para la identificación de conceptos, a fin de realizar una lectura documentaria más rápida y estratégica desde el punto de vista profesional. Es por eso que surgió la idea de elaborar un modelo de lectura que utilizara el conocimiento de estructuras textuales del lector apoyado en estrategias más sistemáticas de identificación de conceptos para agilizar su comprensión y realizar una lectura rápida, en la que el indiza- 
dor se vuelve más estratégico que el lector normal (Fujita, 2003). Por lo tanto, el Modelo de Lectura Documentaria fue propuesto por Fujita (2003b) a consecuencia de los resultados obtenidos en investigaciones de abordaje cognitivo realizadas con Protocolo Verbal (2), de los conocimientos procesales, declarativos y metacognitivos de indizadores en cuanto realizaban la tarea de indexación de artículos científicos. La concepción del modelo de lectura se basó en el ensayo teórico, a partir de los fundamentos proporcionados por los estudios de estructura textual, y la existencia de propuestas de modelos de lectura, fundamentos del análisis conceptual del PRECIS y el abordaje sistemático de la Norma 12.676.

\begin{tabular}{|c|c|c|}
\hline $\begin{array}{l}\text { Concepto } \\
\text { (análisis conceptual) }\end{array}$ & $\begin{array}{l}\text { Cuestionamiento } \\
\text { (norma 12.676) }\end{array}$ & $\begin{array}{l}\text { Parte de la } \\
\text { estructura textual }\end{array}$ \\
\hline Objeto & $\begin{array}{l}\text { ¿El documento posee } \\
\text { en su contexto un } \\
\text { objeto sobre efecto de } \\
\text { una actividad? }\end{array}$ & $\begin{array}{l}\text { Introducción } \\
\text { (objetivos) }\end{array}$ \\
\hline Acción & $\begin{array}{l}\text { ¿El asunto contiene un } \\
\text { concepto activo (por } \\
\text { ejemplo, una acción, } \\
\text { una operación, un } \\
\text { proceso, etc.)? }\end{array}$ & $\begin{array}{l}\text { Introducción } \\
\text { (objetivos) }\end{array}$ \\
\hline Agente & $\begin{array}{l}\text { ¿El documento posee } \\
\text { un agente que practicó } \\
\text { esta acción? }\end{array}$ & $\begin{array}{l}\text { Introducción } \\
\text { (objetivos) }\end{array}$ \\
\hline Métodos del agente & $\begin{array}{l}\text { ¿Este agente se refiere } \\
\text { a modos específicos } \\
\text { para realizar la acción } \\
\text { (por ejemplo, } \\
\text { instrumentos } \\
\text { especiales, técnicas o } \\
\text { métodos)? }\end{array}$ & Metodología \\
\hline Local o ambiente & $\begin{array}{l}\text { ¿Todos estos factores } \\
\text { son considerados en el } \\
\text { contexto de un lugar } \\
\text { específico o ambiente? }\end{array}$ & Metodología \\
\hline Causa y efecto & $\begin{array}{l}\text { ¿Son identificadas } \\
\text { algunas variables } \\
\text { dependientes o } \\
\text { independientes? }\end{array}$ & $\begin{array}{l}\text { Resultados; } \\
\text { discusión de } \\
\text { resultados }\end{array}$ \\
\hline $\begin{array}{l}\text { Punto de vista del } \\
\text { autor; perspectiva }\end{array}$ & $\begin{array}{l}\text { ¿El asunto fue } \\
\text { considerado desde un } \\
\text { punto de vista, } \\
\text { normalmente no } \\
\text { asociado con el campo } \\
\text { de estudio (por } \\
\text { ejemplo, un estudio } \\
\text { sociológico o } \\
\text { religioso)? }\end{array}$ & Conclusiones \\
\hline
\end{tabular}

Tabla I. Modelo de Lectura Documentaria para textos científicos: identificación de conceptos por cuestionamiento en partes de la estructura textual (Fuente: Fujita, 2003, p. 202).

El Modelo de Lectura Documentaria está dispuesto en la tabla 1 que combina las sistemáticas de identificación de conceptos por el análisis conceptual (primera columna) y el abordaje sistemático por el cuestionamiento de la Norma 12.676 (segunda columna) con la localización de los conceptos en parte de la estructura textual (tercera columna).

El modelo de lectura documentaria, elaborado y aplicado, trajo importantes contribuciones para el desenvolvimiento y obtención de herramientas para el Programa de orientación a la formación del indizador, destacándose su fundamento teórico y los resultados del análisis de concepciones en los protocolos de los sujetos que participaron de la evaluación experimental.

La primera aplicación del modelo de lectura fuera del ámbito del Proyecto de Investigación con la intención de probar su divulgación y aplicabilidad fue realizada con profesores de indexación durante el Encuentro Nacional de Investigación en Ciencia de Información de Londrina en octubre de 2002. Los resultados obtenidos, revelaron la aceptación de la metodología con las siguientes observaciones para la práctica del indizador:

- Necesidad de uso de diccionarios especializados del área del texto para verificar la descripción de determinados términos.

- El modelo se aplica a los artículos con determinada estructura textual que no presenten fallas, en caso contrario, existirán dificultades para seguir la estructura del modelo de lectura.

- La selección de términos no podrá ser ejecutada sin vinculación del indizador con una política de indexación.

- El uso del modelo de lectura debe ser vinculado a la formación inicial del indizador y tornarse una práctica constante, pues existirán dificultades en seguir la estructura del modelo de lectura en caso que el indizador ya tenga otra sistemática de indexación y poco contacto con el modelo.

En el año 2006, la propuesta del modelo de lectura documentaria fue aplicada en la disciplina de graduación "Lectura Documentaria", así como también en la educación continuada de bibliotecarios que realizan actividades de análisis de asunto para catalogación en sistemas de bibliotecas universitarias.

En la evaluación del uso del referido Modelo, los bibliotecarios comentaron que son más capaces de realizar actividades de indexación con el Modelo de Lectura Documentaria desde que lo utilizan más intensamente. Por ello, recomiendan que su metodología sea completamente adoptada en la práctica cotidiana de indexación en su actuación profesional. Por otro lado, lamentaron el hecho de no tener, durante la graduación, la oportunidad de una formación inicial en indexación mediante el Modelo de Lectura Documenta- 
ria, motivo por el cual recomiendan su inclusión en los contenidos disciplinares con el acompañamiento del profesor en la mediación del aprendizaje.

El Modelo de Lectura Documentaria para indexación de textos científicos es fundamental para apoyar la propuesta del Programa de orientación para la formación del indizador por el fundamento teórico-metodológico en el cual se basó, y por los resultados obtenidos en las aplicaciones experimentales.

En consecuencia con las recomendaciones acerca del Programa de Orientación a la Formación del Indizador (fujita, 2003), indicamos la necesidad de presentar a los Cursos de Graduación una propuesta de adecuación y/o inclusión del contenido programático, metodologías de enseñanza y bibliografía que se refieran a la lectura documentaria para análisis de asunto.

\section{C) Recursos pedagógicos para un abordaje cognitivo y socio-cognitivo}

La dificultad del indizador frente a la complejidad del análisis de asunto de documentos es el punto de partida que motivó los estudios para el desarrollo del modelo de lectura documentaria, que propone una metodología basada en el uso de estrategias de lectura.

El indizador es un lector con conocimiento previo lingüístico, textual, del mundo profesional y, también, específico, en el caso de indizadores especialistas. Las dificultades existen porque la lectura es un proceso de interacción con el texto escrito que pretende su comprensión y eso significa un proceso de cognición. El proceso de análisis de asunto para indexación, de esa forma, envuelve la comprensión del texto mediante procesos cognitivos, realizados con base en esquemas mentales.

Se resalta, de esa forma, que es necesario no solamente adecuar los contenidos programáticos, sino introducir metodologías de aprendizaje del proceso de lectura (Neves 2006a; Fujita 2007), como por ejemplo, con el uso de la técnica de los protocolos verbales en sus diferentes modalidades (Fujita, 2003; Neves, 2006b). La metodología de enseñanza de lectura en sala de aula se basa en las dificultades de lectura presentadas por los alumnos, que deberán conocerlas a partir de un abordaje cognitivo para facilitar la superación de esas dificultades.

De esa manera, la aplicación del Protocolo Verbal en el aula para la tarea de análisis de asunto de un artículo científico tiene la finalidad de concientizar al alumno sobre la complejidad de la tarea de lectura y de que pueda observarse como lector.

La aplicabilidad del Protocolo Verbal, como recurso de aprendizaje en la enseñanza de indexación para indizadores aprendices, puede ser realizada durante el desarrollo de la disciplina "Lectura Documentaria" conforme relata Fujita (2007) para la tarea de análisis de asunto de un artículo científico.

Para la aplicación en el aula, durante la disciplina "Lectura Documentaria", fueron adoptados los procedimientos de colecta de datos con Protocolo Verbal conforme las orientaciones de Fujita, Nardi y Fagundes (2003) y Neves (2006b), sintetizadas a continuación:

- Procedimientos anteriores a la colecta de datos: elección del texto base; definición de la tarea; selección del sujeto; conversación informal con el sujeto; familiarización con la tarea "Think Aloud".

- Procedimientos durante la colecta de datos: grabación del "Pensar alto" durante la lectura del texto-base; entrevista retrospectiva (opcional).

- Procedimientos posteriores a la colecta de los datos: transcripción literal de las grabaciones de las palabras del sujeto; y análisis de los datos constando de la lectura detallada de los datos, en búsqueda de los fenómenos significativos conforme a los "Parámetros para análisis de transcripciones de protocolos verbales".

La observación del proceso de análisis de asunto para la indexación con Protocolo Verbal puede ser realizada de forma autónoma por el alumno, de modo que el mismo acompañe sus procesos cognitivos desarrollados durante la comprensión de la lectura de texto con la finalidad del análisis temático para la identificación y selección de conceptos.

Este procedimiento de auto-análisis de su proceso de comprensión de lectura podrá ser observado mediante el uso del protocolo verbal durante la ejecución de la tarea de análisis. Entendemos que la metodología del Protocolo Verbal debe ser utilizada en el aula como recurso de aprendizaje, en la medida en que el Pensar Alto durante la lectura documentaria para la indexación o elaboración de resúmenes deberá revelar estrategias y dificultades que, conocidas, podrán ser mejor ejecutadas o perfeccionadas.

Al final de la disciplina, se propone la aplicación de la modalidad del Protocolo Verbal en Grupo con la finalidad de discutir el texto en el aula que tenga como contenido la tarea de indexación. 


\section{Disciplina: “Lectura Documentaria \\ Sumario}

Naturaleza del proceso de lectura y uso de estrategias. Diferentes visiones de lectura desde las primeras visiones cognitivistas hasta la visión socio construccionista de lectura como evento social. Protocolo verbal como técnica de colecta de datos de proceso de lectura. El proceso de lectura para análisis de documentos con fines de indexación y resumen: recientes aplicaciones del protocolo verbal.

\section{Objetivos}

Concientizar al alumno sobre la naturaleza del proceso de lectura y el uso de estrategias;

Familiarizar al alumno con las diferentes visiones del proceso de lectura, desde las primeras visiones cognitivistas hasta la visión socio-construccionista de lectura como evento social;

Familiarizar al alumno con el proceso de lectura para Análisis Documentario y con la reciente utilización del protocolo verbal en investigaciones de esa área.

\section{Contenido Programático}

1. Proceso de comprensión de texto: diferentes visiones de lectura

1.1. De proceso linear a la co-enunciación

1.1.1. Lectura y procesamiento de la información

1.1.2. Lectura y adivinación

1.1.3. Lectura y conocimiento previo: lingüístico, textual, de mundo y profesional

1.2. Lectura, planificación, metacognición y estrategias

1.3. El proceso interactivo entre las variables de la lectura: el texto, el lector y el contexto

2. Introspección y observación de proceso de lectura

2.3. Protocolo verbal como instrumento de colecta de datos

2.3.3. Adaptaciones del protocolo verbal (Protocolo con pausa / Protocolo con entrevista retrospectiva /

Protocolo interactivo / Protocolo en grupo o evento social de lectura)

3. Lectura documentaria: aspectos profesionales

3.1. Lectura en análisis documentaria: interacción de las variables

3.1.1. El lector innato y profesional: formación y capacitación

3.1.2. El texto: tipologías, superestructura y macroestrutura textuales

3.1.3. El contexto: el sistema de información, la política de análisis documentaria y lenguaje documentario

3.2. El proceso de análisis de asunto por la identificación y selección de conceptos: concepciones y procedimientos en la lectura

4. Estrategias de lectura en Análisis Documentaria para comprensión del contenido y objetivos de la demanda: estudios de observación

5. Proceso de lectura para análisis documentaria: proposición metodológica.

Tabla II. Plan de enseñanza de la disciplina "Lectura Documentaria"

\section{La enseñanza de la lectura documentaria para la indexación: aspectos conceptuales y metodológicos del plan de enseñanza}

La propuesta fue formulada (Fujita, 2003) por medio de un plan de enseñanza que contiene la parte conceptual y la parte metodológica de las Directrices del Programa de Orientación. En esta propuesta del plan de enseñanza se destacan: el sumario, los objetivos y el contenido programático. El sumario prioriza la lectura como proceso en el abordaje cognitivo y socio-cognitivo con la indicación del uso del Protocolo Verbal como recurso pedagógico y la lectura vista en la perspectiva del análisis documentario para la indexación y elaboración de resúmenes. Los objetivos son claros y expresan la necesidad de la enseñanza de la lectura documentaria para la concientización del alumno como lector en el abordaje cognitivo hasta el esclarecimiento del proceso de lectura durante el Análisis Documentario.

El plan de enseñanza de la disciplina "Lectura Documentaria" inicia la secuencia de contenido con la propuesta de revelar al alumno que él es un lector, haciendo que tome consciencia sobre la naturaleza del proceso de lectura y el uso de las diferentes estrategias (ítem 1). Para que el alumno pueda observar a sí mismo en la actividad de lectura y verificar qué estrategias utiliza en la lectura, se le presentará la técnica de colecta de datos introspectiva en las diferentes modalidades del Protocolo Verbal (item 2). En el item 3 el alumno constata que necesita convertirse en un lector profesional teniendo en cuenta las variables de lectura documentaria, el lector, el texto y el contexto, que estarán condicionadas a la especificidad de las operaciones de análisis documentario: indexación, clasificación y elaboración de resúmenes. En seguida, serán discutidas las operaciones de Análisis Documentario en lo que respecta a la identificación y selección de conceptos para la representación de contenidos documentarios (item 3.2) y las estrategias de lectura documentaria en Análisis Documentario observadas en las colectas de datos introspectivos con indizadores de sistemas de información. El último ítem presenta el Modelo de Lectura Documentaria para la indexación de textos científicos y su Manual de Enseñanza como recurso pedagógico (item 5 ).

\section{Consideraciones finales}

La disciplina "Lectura Documentaria" forma parte del contenido Curricular del Curso de Biblioteconomía de la UNESP - Campus Marília, desde 2004, con contenido dedicado a la con- 
cientización del lector profesional de Ciencia de Información para la lectura documentaria.

En la disciplina "Lectura Documentaria", fue adoptado el plan de enseñanza recomendado por las directrices del Programa, acrecentado con un plan de aulas que permite la práctica pedagógica de la lectura documentaria mediante su fundamentación teórica y el uso del Protocolo Verbal para la observación, por el alumno, del proceso cognitivo de lectura documentaria.

El contenido de la disciplina "Lectura Documentaria" debe dar énfasis al conocimiento de la lectura y sus estrategias, de modo que prepare al alumno para los contenidos de las actividades de indexación, clasificación y elaboración de resúmenes.

Con el desarrollo de la disciplina, constatamos que el indizador aprendiz necesita prever el contexto profesional real y desarrollar conocimientos y estrategias profesionales adecuadas al análisis de asunto. El modelo de lectura trae esta contribución, facilitando el proceso de indexación, además de mostrarse una herramienta de auxilio, pues orienta a los indizadores aprendices sobre lo que deben buscar y dónde encontrarlo.

El protocolo verbal individual, en el cual el sujeto exterioriza sus procesos mentales en cuanto la información procesada está bajo el foco de su atención, proporcionó una mejor observación del proceso de lectura por la verbalización del conocimiento procesal, lo que recomienda su uso como recurso de aprendizaje. La investigación concluye que la enseñanza de la indexación se perfecciona teniendo como referencia la auto-observación, con Protocolo Verbal, del procesamiento mental realizado en la lectura documentaria, así como la concientización de los docentes de la necesidad de capacitación como indizadores experimentados.

Después del desarrollo del contenido de la disciplina "Lectura Documentaria", en el 2006, se observó disminución de las dificultades de análisis de asunto practicada en las disciplinas "Indexación" y "Condensación documentaria: elaboración de Resúmenes", lo que prueba la necesidad de concientizarse sobre la importancia de las estrategias de lectura documentaria en la formación del indizador.

\section{Notas}

(1) Mariângela Spotti Lopes Fujita: Profesora Adjunta del Departamento de Ciencia de Información de la Facultad de Filosofía y Ciencias de la Universidad Estadual Paulista - UNESP, Campus de Marília Brasil.
(2) La técnica del protocolo verbal consiste en analizar todo proceso de verbalización del participante en cuanto realiza su actividad con el mínimo de interacción con el investigador. Esta exteriorización es grabada y transcrita literalmente, produciendo protocolos verbales. Los protocolos son generalmente definidos como relatos verbales de los procesos mentales conscientes de los informantes" (Fujita, 2005).

\section{Referencias}

Fujita, M. S. L. (2004). A leitura documentária na perspectiva de suas variáveis: leitor-texto-contexto. // Datagramazero Revista de Ciência da Informação. Rio de Janeiro. 5: 4 (2004). http://www.dgz.org.br. (2006-06-10).

Fujita, M. S. L. (2007). A abordagem cognitiva da leitura como prática pedagógica no ensino da disciplina leitura documentária no curso de Biblioteconomia da UNESP Campus de Marília: uso do protocolo verbal para metacognição do indexador aprendiz. // Santos, Jussara Pereira Santos (org.). A leitura com prática pedagógica na formação do profissional da informação. Rio de Janeiro: Fundação Biblioteca Nacional, 2007. 101-132.

Fujita, M. S. L., Nardi, M. I. A., Fagundes, S. A. (2003). A observação da leitura documentária por meio de protocolo verbal. // Rodrigues, G. M., Lopes, I. L. (org.). Organização e representação do conhecimento na perspectiva da ciência da Informação. Brasília: Thesaurus, 2003. 141-178. (Estudos Avançados em Ciência da Informação, v. 2).

Fujita, M. S. L. (2003). A leitura documentária do indexador: aspectos cognitivos e lingüísticos influentes na formação do leitor profissional. 2003. Marília: Faculdade de Filosofia e Ciências, UNESP, 2003. Tese de Livre-Docência em Análise Documentária e Linguagens Documentárias Alfabéticas.

Giasson, J. (1993). A compreensão na leitura. Lisboa: Asa, 1993.

Hjørland, B. (2002). Epistemology and the sócio-cognitive perspective in information science. // Journal of the American Society for Information Science and Technology. 53: 4 (2002) 257-70.

Nardi, M. I. A. (1993). As expressões metafóricas na compreensão do texto escrito em língua estrangeira. São Paulo: Pontifícia Universidade Católica, 1993. Dissertação de Mestrado.

Nardi, M. I. A. (1999). A metáfora e a prática de leitura como evento social: instrumentos do pensar a biblioteconomia do futuro. São Paulo: Pontifícia Universidade Católica de São Paulo, 1999. Tese de Doutorado.

Neves, D. A. de B. (2006a). Ciência da informação e cognição humana: uma abordagem do processamento da informação. // Ciência da Informação. Brasilia. 35:1 (2006a). http://www.scielo.br/scielo .php/script_sci_se rial/pid_0100-1965/lng_pt/nrm_ iso. (2007-05-10).

Neves, D. A. de B. (2006b). La verbalización como registro para análisis en la investigación sobre lectura. // Anales de Documentación. 9 (2006b) 43-51. 